\title{
Anaesthetic management of patients undergoing surgery for Moyamoya disease - our institutional experience
}

\author{
Vivek B. Sharma, Hemanshu Prabhakar, Girija Prasad Rath, Parmod K. Bithal
}

\begin{abstract}
Background: Moyamoya disease (MMD) is a chronic cerebrovascular disorder, defined as the progressive stenosis or occlusion of the intracranial vessels. Because of the insecure cerebral circulation, these patients represent an anaesthetic challenge. Literature is scarce on the anaesthetic management of MMD, especially from the Indian subcontinent. The main objective of our study was to evaluate the peri-operative course and outcome of patients undergoing surgery for MMD. Materials and Methods: We analysed available medical records of all patients who underwent revascularisation surgeries for MMD over a period of 10 years (January 2002 to June 20I2).Various intra- and post-operative data related to anaesthesia were recorded. The patients for the analysis were divided into two groups: Group I, paediatrics ( $<18$ years) and group 2, adults ( $\geq 18$ years). Data are presented as number (\%) or mean \pm SD or median (range). Results: There were 36 patients ( 12 adults and 24 children). Normotension, normovolaemia, normo- or mild-hypercapnia and normothermia were maintained in all patients. No patient developed complications or new neurological deficit in the post-operative period. All patients were discharged with a full Glasgow coma scale (GCS) and no new neurologic deficit. Conclusion: Although the incidence of MMD is not high in India, it is an important cause of cerebral stroke in children and adults. Proper pre-operative evaluation is the most effective method to achieve good results. Anaesthetic management of MMD should focus on the maintenance of adequate cerebral blood flow and cerebral perfusion pressure ensuring adequate cerebral oxygenation to avoid ischaemic complications.
\end{abstract}

Key words: Anaesthesia, moyamoya disease, risk factors, surgery

\section{INTRODUCTION}

Moyamoya disease (MMD) is a chronic cerebrovascular disorder, defined as the progressive stenosis or occlusion of the intracranial vessels. The stenosis begins from the terminal bifurcation of internal carotid arteries (ICAs) and gradually progresses to the anterior, middle and posterior cerebral arteries. The stenosis of these arteries is accompanied by a collateral network of vessels formed

\begin{tabular}{|l|l|}
\hline \multicolumn{2}{|c|}{ Access this article online } \\
\hline Quick Response Code: & Website: \\
\hline & www.jnaccjournal.org \\
\cline { 2 - 2 } & \\
\hline
\end{tabular}

at the base of the brain, which produces the characteristic reticulate appearance "puff of smoke" on angiography. MMD was first described by Takeuchi and Shimuzui in 1957. ${ }^{[1]}$ Moyamoya, a Japanese word meaning 'something hazy like a puff of cigarette smoke drifting in the air', [Figure 1] is the angiographic appearance of abnormal collateral vasculature. ${ }^{[2]}$

Although the pathogenesis of the disease remains unknown, it is probably a genetically inherited disease, as a familial occurrence is reported in $9-12 \%$ of cases. ${ }^{[3]}$

The main pathological findings in MMD are fibrocellular thickening of the intima, irregular undulation of the internal elastic laminae and attenuation of the media. ${ }^{[4]}$ MMD can cause a range of symptoms including ischaemic stroke, transient ischaemic attack (TIA), intracranial haemorrhage, seizures and headaches. ${ }^{[5]}$

Department of Neuroanaesthesiology, Neurosciences Center, All India Institute of Medical Sciences, New Delhi, India

Address for correspondence:

Dr. Hemanshu Prabhakar, Department of Neuroanaesthesiology, Neurosciences Center, All India Institute of Medical Sciences, New Delhi - 110029 , India. E-mail: prabhakarhemanshu@rediffmail.com 
MMD or cerebral infarction due to the occlusion or stenosis of the ICA and intracranial bleeding due to dilated, fragile collateral vessels as compensatory response to ischaemia.

Increasingly, these patients present for surgical management to improve the cerebral circulation. Patients with recurrent or progressive cerebral ischaemic events and reduced cerebral perfusion are candidates for surgery. Out of all the treatment options, revascularisation surgery is the most successful therapy in preventing stroke for MMD patients. ${ }^{[5]}$ Surgical revascularisation procedures have been shown to provide symptomatic benefit in $87 \%$ of patients. ${ }^{[6]}$ The anaesthetic management of these patients has evolved over the years with an increased understanding of the disease. These have specifically resulted from the identification of risk factors for peri-operative complications and outcomes related to the use of anaesthetic agents. Because of the insecure cerebral circulation, these patients represent an anaesthetic challenge.

There are no prospective or retrospective studies on the anaesthetic management of MMD in India except few isolated case reports. The main objective of our study was to evaluate the peri-operative course and outcome of patients of MMD anaesthetised for revascularisation procedure.

\section{MATERIALS AND METHODS}

After approval from Institute Ethics Committee, we analysed available medical records of all patients who underwent re-vascularisation surgeries for MMD over a period of 10 years (January 2002 to June 2012). For each patient, data was collected by review of records related to clinical presentation, diagnosis, surgical procedure, intra-operative course and post-operative outcome at discharge. Pre-operative data included age, weight, gender, presenting complaints, ischaemic or haemorrhagic presentation on non-contrast computed tomography head, unilateral or bilateral disease on digital subtraction angiography (DSA).

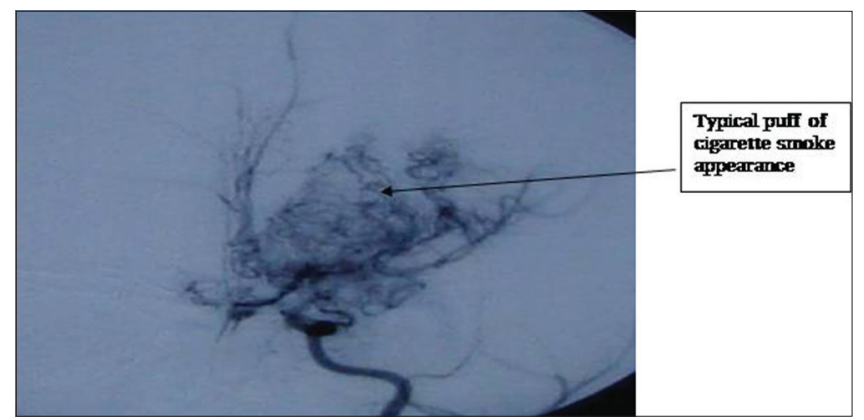

Figure 1: This digital subtraction angiography film shows the typical puff of smoke appearance representing tortuous basal 'Moyamoya'collaterals
Intra-operative data included anaesthetic technique, fluids infused, urine output and blood loss/transfusion. Intra-operative complications such as cardiovascular, respiratory (bronchospasm, laryngospasm, endobronchial intubation and pneumothorax) and others (inadequate recovery from neuromuscular blockade, hypothermia $\left[<35^{\circ} \mathrm{C}\right]$, hyperthermia $\left[>38^{\circ} \mathrm{C}\right]$ ) were also recorded. Post-operative parameters included duration of mechanical ventilation, re-intubation, fever, hydrocephalus, seizures, meningitis and any other surgical complications, duration of intensive care unit/ hospital stay and neurologic status at discharge (Glasgow coma scale; GCS). The patients for the analysis were divided into two groups: group 1 , paediatrics ( $<18$ years) and group 2 , adults ( $\geq 18$ years).

Data are presented as number (\%) or mean \pm SD or median (range). Statistical analysis was done using SPSS version 15.0.

\section{RESULTS}

We analysed the records of 39 patients with MMD who underwent surgical re-vascularisation procedures at our institute. The medical records of three patients were incomplete and were excluded from the study. Finally, the medical records of 36 patients were analysed. Demographic data are shown in Table 1 . The presenting features are tabulated [Table 2].

As per our institutional protocol in MMD patients, pre-medication for anxiolysis was avoided in all the patients including children. Glycopyrrolate $0.2 \mathrm{mg}$ intramuscular before shifting to the operation theatre was given in adults and Atropine $30 \mu \mathrm{g} / \mathrm{kg}$ in children was given orally 30-45 min before surgery.

In the operation theatre, after applying monitoring in form of heart rate, electrocardiogram (ECG), non-invasive blood pressure, pulse oxymetry, induction was achieved

Table 1: Demographic profile, presentation and diagnostic data (mean $\pm \mathrm{SD} / n(\%) /$ median [range])

\begin{tabular}{lcc}
\hline Variables & $\begin{array}{c}\text { Group 1 } \\
\text { paediatric } \\
\text { (age } \leq \mathbf{1 8} \text { years) }\end{array}$ & $\begin{array}{c}\text { Group 2 } \\
\text { adults } \\
\text { (age } \geq \mathbf{1 8} \text { years) }\end{array}$ \\
\hline Number of patients & 24 & 12 \\
Age (years) & $3-16(9)$ & $19-52(32)$ \\
Weight (kg) & $12-55(22)$ & $41-85(65)$ \\
Gender (male:female) & $11: 13$ & $7: 5$ \\
Ischaemic: & $22(91.1 \%):$ & $3(25 \%):$ \\
haemorrhagic disease & $2(8.06 \%)$ & $9(75 \%)$ \\
Unilateral: Bilateral & $4: 20$ & $1: 11$ \\
disease & & \\
\hline$n=$ Number of patients & &
\end{tabular}


with inhalation (sevoflurane) or intravenous (IV) techniques. Induction in children was most commonly achieved with inhalational method. IV induction was the method of choice in adults but also used in children who had an IV cannula in situ. Fentanyl was given to all patients at the time of induction $2 \mu \mathrm{g} / \mathrm{kg} \mathrm{IV}$, followed by lidocaine $1-1.5 \mathrm{mg} / \mathrm{kg}$ and propofol $1.5-2.0 \mathrm{mg} / \mathrm{kg}$ and rocuronium $0.6-1 \mathrm{mg} / \mathrm{kg}$ to facilitate tracheal intubation.

All patients were placed in the supine position with the head turned to one side to allow surgical access. Intra-arterial catheters were placed in either a radial or dorsalis pedis artery for continuous blood pressure recording. End tidal $\mathrm{CO}_{2}\left(\mathrm{ETCO}_{2}\right)$ was continuously monitored. $\mathrm{ETCO}_{2}$ was maintained between 35 and $40 \mathrm{mmHg}$ to avoid detrimental effects of both hypo- and hyper-capnia. Nasopharyngeal temperature was monitored and forced air warming blankets were used on all patients to maintain normothermia. All patients were mechanically ventilated. Central venous pressure monitoring was only carried out in patients who underwent direct revascularisation bypass procedures. Anaesthesia was maintained with sevoflurane 0.6-0.9 minimum alveolar concentration (MAC) or isoflurane 0.6-0.8 MAC and a nitrous oxide/oxygen mixture or an air/oxygen mixture $\left(\mathrm{FiO}_{2}\right.$ of $\left.0.35-0.5\right)$, with intermittent doses of vecuronium and fentanyl. Adequate warm crystalloids and colloids were administered according to urine output. All patients were catheterised for urine output monitoring. At the end of surgery, the residual neuromuscular block was reversed with neostigmine and glycopyrrolate and the trachea was extubated. All patients were shifted to the intensive care unit after extubation.

The various surgeries carried out in these patients are shown in Table 3. The peri-operative data and intensive care unit (ICU) and hospital stay are shown in Table 4.

Intra-operative, two patients in paediatric age group and three patients in adult group developed hypertension at the time of scalp incision and raising of bone flap, which was promptly managed with labetalol 5-10 mg boluses. It was found that one patient in paediatric age group and two in adult group developed hypotension, one after bone-flap removal and two immediately after anaesthesia induction. All patients were treated with mephentramine sulphate. One patient in paediatric age group who had delayed awakening was elective ventilated to the ICU and extubated after $5 \mathrm{~h}$ of observation. No patient developed any complications or new neurological deficit in the post-operative period. All patients were discharged with a full GCS and no new neurologic deficit.

\section{DISCUSSION}

In 2003, a nationwide epidemiological survey in Japan showed a prevalence and incidence of MMD as 6.03
Table 2: Presenting features $[n(\%)]$

\begin{tabular}{lcc}
\hline Presentation & $\begin{array}{c}\text { Paediatric } \\
(\boldsymbol{n}=\mathbf{2 4}) \mathbf{( \% )}\end{array}$ & $\begin{array}{c}\text { Adults } \\
(\boldsymbol{n}=\mathbf{1 2}) \mathbf{( \% )}\end{array}$ \\
\hline Fever & $2(12.5)$ & 0 \\
Loss of consciousness & 0 & $2(16.7)$ \\
Headache & $2(8.3)$ & $7(58.3)$ \\
Seizures & $5(20.8)$ & $1(8.3)$ \\
Neurological deficit & $20(83.3)$ & $9(75)$ \\
Ischaemic stroke & $19(79.2)$ & $3(16.7)$ \\
Haemorrhage & $2(8.3)$ & $9(66.7)$ \\
\hline$n=$ Number of patients & &
\end{tabular}

Table 3: Surgical treatment procedures for patients with moyamoya disease $(n)$

\begin{tabular}{lc}
\hline Direct re-vascularisation & $n=10$ \\
STA-MCA Anastomosis & 3 \\
STA-MCA Anastomosis + EDAMS & 6 \\
STA-MCA Anastomosis + EDAS & 1 \\
Indirect re-vascularisation & $n=26$ \\
EDAMS & 24 \\
EMS & 1 \\
EDAS & 1 \\
\hline
\end{tabular}

$n=$ Number of patients, STA $=$ Superficial temporal artery, MCA $=$ Middle cerebral artery, EDAMS = Encephalo-duro-arterio-myo-synang-iosis, EMS $=$ Encephalo-myo-synangiosis, EDAS $=$ Encephalo-duro-arteriosynangiosis

Table 4: Baseline Haemoglobin, baseline Haematocrit, intra-operative data, ICU stay, hospital stay (mean $\pm \mathrm{SD} / n(\%) /$ median [range])

\begin{tabular}{lcc}
\hline & $\begin{array}{c}\text { Paediatric } \\
\text { group } \\
(\boldsymbol{n}=\mathbf{2 4 )}\end{array}$ & $\begin{array}{c}\text { Adult } \\
\text { group } \\
(\boldsymbol{n}=\mathbf{1 2})\end{array}$ \\
\hline Haemoglobin (g\%) & $11.52 \pm 0.76$ & $12.95 \pm 1.67$ \\
Haematocrit & $34.60 \pm 2.25$ & $38.85 \pm 5.03$ \\
Duration of surgery (h) & $3.49 \pm 1.53$ & $4.50 \pm 1.20$ \\
Duration of anaesthesia (h) & $4.39 \pm 1.72$ & $5.39 \pm 1.19$ \\
Intra-operative fluids (ml) & $1098 \pm 751.4$ & $2566 \pm 1034.2$ \\
Urine output (ml) & $294 \pm 271.6$ & $779.17 \pm 549.9$ \\
Blood loss (ml) & $88.75 \pm 54.3$ & $150 \pm 67.72$ \\
ICU stay (days) & $1.50 \pm 0.72$ & $1.42 \pm 0.66$ \\
Hospital stay (days) & $12(7-24)$ & $14(9-21)$ \\
\hline ICU = Intensive care unit & &
\end{tabular}

ICU = Intensive care unit

and 0.54 per 100000 , respectively. ${ }^{[7]}$ There has been no nationwide epidemiological survey of the prevalence and incidence of MMD in other Asian countries except a regional epidemiological survey in China. ${ }^{[8]}$

We have reviewed our experience with 36 patients who underwent various revascularisation neurosurgical procedures for MMD. The clinical manifestations 
of MMD have strong age-related and geographic differences. Classically, two distinct age groups are affected: children between 5 and 7 years and young adults in their mid-thirties (up to 40 years). The clinical presentation is variable, but is essentially that of cerebrovascular insufficiency, resulting in focal motor and sensory disturbances. In children, manifests commonly as ischaemic stroke, paroxysmal hemiplegia is a common presenting symptom, while in adults a sudden loss of consciousness associated with cerebral haemorrhage, most frequently in basal ganglia. ${ }^{[9]}$ Subarachnoid or intra-ventricular haemorrhage may also be observed. In our study, children commonly presented with ischaemic stroke, TIA and headache, whereas intracranial haemorrhage was rare and adults presented mainly with haemorrhagic symptoms.

The goal for anaesthetic management is to maintain balance between oxygen supply and demand. In MMD it becomes more important to avoid any further neurological deterioration because of the underlying insecure and tenous cerebral vasculature. Cerebral blood flow (CBF) is maintained by avoiding hypotension and maintaining normocarbia while appropriate depth of anaesthesia and adequate analgesia is used for minimising and prevention of increase in cerebral oxygen consumption $\left(\mathrm{CMRO}_{2}\right)$ associated with pin application, laryngoscopy, tracheal intubation and surgical stimulus. ${ }^{[5]}$

A decreased haematocrit owing to anaemia or peri-operative blood loss places MMD patients at the risk of cerebral ischaemia. An ideal haematocrit is debatable, but a haematocrit of $30-42 \%$ has been proposed as adequate. ${ }^{[10,11]}$

Pre-medication for anxiolysis may be beneficial in children with MMD as crying causes hyperventilation resulting in hypocapnia with possible cerebral vasoconstriction, decreased CBF and cerebral ischaemia. ${ }^{[12]}$ There are no evidence-based data suggesting any advantage or better outcome with any particular premedication although midazolam is most commonly used. ${ }^{[12]}$

Hyperventilation has been identified as a factor that may induce ischaemic symptoms in Moyamoya patients. In a study, Matsushima et al., investigated 169 encephalo-duro-arterio-myo-synang-iosis (EDAS) procedures in children and found that $6(3.6 \%)$ of the patients developed cerebral infarction as a result of hyperventilation associated with crying. ${ }^{[13]}$ It is extremely important to prevent peri-operative crying in these cases. This requires proper pre-medication, smooth inhalational or IV induction, and good post-operative pain management. Adequate oral sedation should be administered pre-operatively; however, it should always be kept in mind that excessive sedation can induce hypercapnia, which is also a known risk factor for ischaemic complications. When securing venous access before and during anaesthesia induction, pain can be minimised by applying topical anaesthetic creams. The basic principle in the anaesthetic management should be the preservation of a proper balance between cerebral blood flow and oxygen consumption. No evidence exists, which can prove the association of use of a particular anaesthesia technique or drug with better outcome. The goal of the anaesthetic induction was therefore to maintain stable systemic as well as cerebral haemodynamics. The preferred method for anaesthesia induction in MMD is with IV agents taking care of blood pressures. If hypotension occurs during induction or maintenance, vasoconstrictors should be administered immediately. Sevoflurane is the alternative. Induction was achieved in our study with inhalation agent, sevoflurane, in small children. Sevoflurane provides better recovery compared with isoflurane in children. ${ }^{[13,14]}$ In older children, who had an IV cannula already in place and in adults, IV induction was carried out using graded doses of propofol. A non-depolarising muscle relaxant, which does not cause cardiovascular change or histamine release and vasodilatation, such as vecuronium, is also suitable for smooth intubation and for maintaining muscle relaxation throughout the surgery. The haemodynamic pressor response at intubation was attenuated by use of lignocaine, fentanyl or an additional bolus dose of propofol.

Anaesthesia may be maintained with a volatile agent and opioids or with total intravenous anaesthesia (TIVA) using propofol and opioids with or without a low dose of volatile agent. The use of nitrous oxide is controversial. It has been used in patients undergoing re-vascularisation procedures and during cardio-pulmonary bypass in patients with MMD. ${ }^{[15,16]}$ Sakamoto et al., retrospectively analysed $368 \mathrm{MMD}$ cases for re-vascularisation surgery in whom nitrous oxide was used and found that the severity of the disease and type of surgical procedure were major determinants of post-operative neurological deterioration rather than anaesthetic management that included nitrous oxide. ${ }^{[16]}$ Sato et al., studied the effect of inhalation anaesthesia and TIVA on cerebral circulation in MMD and measured regional cortical blood flow ( $\mathrm{rCoBF})$, jugular bulb oxygen saturation and frontal regional oxygen saturation by near-infrared spectroscopy in patients undergoing re-vascularisation procedures. The investigators concluded that regional $\mathrm{rCoBF}$ levels may be decreased with inhalation anaesthesia and may provoke intra-cerebral steal, which was not seen with TIVA. ${ }^{[17]}$ Adachi et al., compared the effect of sevoflurane and propofol on intracranial pressure (ICP) and $\mathrm{rCoBF}$ using a laser Doppler flow meter probe during revascularisation procedures in patients with MMD and concluded that rCoBF was significantly higher and ICP lower with propofol. ${ }^{[18]}$ 
During general anaesthesia, hyperventilation and the resultant hypocapnia may reduce CBF and thus precipitate ischaemic symptoms. In MMD patient's hypercapnia dilates the normal cerebral vessels, whereas the diseased vessels, which are already maximally dilated, show minimal response resulting in less blood flow to areas supplied by diseased vessels. Kurehara et al., ${ }^{[19]}$ measured CoBF continuously by the laser Doppler method to evaluate the effect of hypercapnia on $\mathrm{rCoBF}$ with MMD and concluded that hypercapnia may be detrimental to the cortical circulation. This suggests that normocapnia should be maintained during anaesthesia. Therefore, other authors argue that normocapnia is the optimal state for the cerebral circulation during cerebrovascular reconstruction in MMD. ${ }^{[20,21]}$ Volatile anaesthetics and nitrous oxide can cause cerebral vasodilation, and this may result in intra-cerebral steal. Since thiopentone or propofol are cerebral vasoconstrictors, there is no danger of steal phenomenon occurring. Therefore, balanced anaesthesia and TIVA are considered the most suitable choices for re-vascularisation procedures in MMD. ${ }^{[18]}$ In our centre, balanced anaesthesia technique was used.

In one study of children with this condition, a group with ischaemic complications had significantly lower urine output than a group without these problems. ${ }^{[22]}$ Blood loss during surgery has also been highlighted as a potential contributor to ischaemic complications in patients with MMD. ${ }^{[22]}$ One of the main goals of fluid therapy in the re-vascularisation surgery in our study groups was maintenance of normovolemia.

It is important peri-operatively to maintain the blood pressure at or above the pre-operative baseline and to avoid fall in blood pressure at all costs to avoid decrease in CPP. Reduction in CBF is better tolerated in adults than children who have higher $\mathrm{CMRO}_{2}{ }^{[12]}$ Auto regulatory response to hypotension is significantly less in children compared with adults ${ }^{[5,23]}$ and they are more prone to develop cerebral ischaemia in response to hypotension. Iwama et al., described their experience with ischaemic complications that occurred in 124 paediatric cases of MMD. They found that 21 (16.9\%) of the children developed this problem, and noted significantly higher incidences of peri-operative transient ischaemic attacks in the patients who had intra- and post-operative hypotension compared with those who had no hypotension peri-operatively. ${ }^{[23]}$ In our study, three patients (one child and two adults) developed hypotension under anaesthesia. All patients were treated promptly with mephentramine sulphate to raise the mean arterial pressure. As a result, none suffered cerebral ischaemia in our series.

It is imperative that normothermia be maintained during re-vascularisation surgery. We used heating blankets to maintain normal body temperature throughout surgery and post-operatively. Hyperthermia has to be avoided as concluded by Kurokawa et al. ${ }^{[24]}$ They showed that hyperthermia increases cerebral metabolic rate, which causes an increase in oxygen consumption and possible cerebral ischaemia.

In this series, a sufficient urinary volume $(2.2 \mathrm{ml} / \mathrm{kg} / \mathrm{h})$ was maintained in all patients, even in those who developed complications. However, this level was significantly lower than that in the group of patients without complications $(4.1 \mathrm{ml} / \mathrm{kg} / \mathrm{h})$. This finding suggested that maintaining the blood volume at a level corresponding to normovolaemia may be insufficient and that the blood volume should be kept in a higher range (hypervolaemic). ${ }^{[22]}$ However, no patient developed cerebral ischaemia despite an output of $2.2 \mathrm{ml} / \mathrm{kg} / \mathrm{h}$, which could be due to surgical technique. At the same time, none with urine output $4.1 \mathrm{ml} / \mathrm{kg} / \mathrm{h}$ showed new signs of cerebral ischaemia. Thus it is likely that maintaining hypervolaemia preempts cerebral ischaemia. In this respect, our observations are in agreement with those of Sato and colleagues. ${ }^{[22]}$

Post-operative cerebral infarction may be associated with inadequate pain control. ${ }^{[11]}$ The use of scalp block during epiduro-angio-myo-synangiosis (EDAMS) surgery in children with MMD has been shown to provide calm awakening, better pain relief and possibly reduced post-operative morbidity. ${ }^{[25]}$ In our study, multi-modal analgaesia strategy was used to provide analgaesia (fentanyl and IV paracetamol) in both the groups and per rectal paracetamol as suppositories in children.

Medical interventions included anti-platelet agents (aspirin and ticlopidine), rheologic therapy (pentoxifylline) and calcium channel antagonists (nimodipine), although the efficacy of these treatments has not been proven. We avoided the long-term use of anti-coagulation because of the concern for haemorrhagic stroke.

\section{CONCLUSION}

In conclusion, we retrospectively analysed the peri-operative course of patients with MMD. Although the incidence of MMD is not high in India, it is an important cause of cerebral stroke in children and adults. Proper pre-operative evaluation is the most effective method to achieve good results. The main goals during anaesthesia are maintenance of normotension, normovolaemia, normo- or mild-hypercapnia and normothermia. Early diagnosis and effective management are necessary before any ischaemic event and neurologic impairment occurs. Re-vascularisation surgery remains the only viable option to decrease further ischaemic insult and 
neurologic deterioration. Anaesthetic management of MMD should focus on the maintenance of adequate cerebral blood flow and cerebral perfusion pressure ensuring adequate cerebral oxygenation to avoid ischaemic complications.

\section{REFERENCES}

1. Takeuchi K, Shimizu K. Hypo genesis of bilateral internal carotid arteries. No To Shinkei. 1957;9:37-43.

2. Suzuki J, Takaka A. Cerebrovascular "moyamoya" disease: Diseases showing abnormal net-like vessels in base of brain. Arch Neurol 1969;20:288-99.

3. Fukuyama Y, Kanai N, Osawa M. Clinical genetic analysis on the moyamoya disease. The research committee on spontaneous occlusion of the circle of willis (moyamoya disease) of the ministry of health and welfare Japan: Annual Report 1992. Tokyo, Japan: Ministry of Health and Welfare Japan; 1992:141-6.

4. Scott RM, Smith ER. Moyamoya disease and moyamoya syndrome. N Engl J Med 2009;360:1226-37.

5. Parray T, Martin TW, Siddiqui S. Moyamoya Disease: A review of the disease and anesthetic management. J Neurosurg Anesthesiol 2011;23:100-9.

6. Fung L, Thompson D, Ganesan V. Revascularisation surgery for peadiatric moyamoya: A review of the literature. Childs Nerv Syst 2005;21:358-64.

7. Kuriyama S, Kusaka Y, Fujimura M, Wakai K, Tamakoshi A, Hashimoto $\mathrm{S}$, et al. Prevalence and clinicoepidemiological features of moyamoya disease in Japan: Findings from a nationwide epidemiological survey. Stroke 2008;39:42-7.

8. Miao W, Zhao PL, Zhang YS, Liu HY, Chang Y, Ma J, et al. Epidemiological and clinical features of Moyamoya disease in Nanjing, China. Clin Neurol Neurosurg 2010;112:199-203.

9. Ikezaki K, Han DH, Kawano T, Kinukawa N, Fukui M. A clinical comparison of definite moyamoya diseases between South Korea and Japan. Stroke 1997;28:2513-7.

10. Lee SH, Heros RC, Mullan JC, Korosue K. Optimum degree of hemodilution for brain protection in a canine model of focal cerebral ischemia. J Neurosurg 1994;80:469-75.

11. Kansha M, Irita K, Takahashi S, Matsushima T. Anesthetic management of children with moyamoya disease. Clin Neurol Neurosurg 1997;99:S110-3.

12. Nomura S, Kashiwagi S, Uetsuka S, Uchida T, Kubota H, Ito $\mathrm{H}$. Perioperative management protocols for children with moyamoya disease. Childs Nerv Syst 2001;17:270-4.

13. Matsushima Y, Aoyagi M, Suzuki R, Tabata H, Ohno K. Perioperative complications of encephalo-duro-arteriosynangiosis: Prevention and treatment. Surg Neurol 1991;36:343-53.
14. Soriano SG, Sethna NF, Scott RM. Anesthetic management of children with moyamoya syndrome. Anesth Analg 1993;77:1066-70.

15. Singh D, Rath GP, Dash HH, Bithal PK. Sevoflurane provides better recovery as compared with isoflurane in children undergoing spinal surgery. J Neurosurg Anesthesiol 2009;21:202-6.

16. Sakamoto T, Kawaguchi M, Kurehara K, Kitaguchi K, Furaya H, Karasawa J. Risk factors for neurologic deterioration after revascularization surgery in patients with moyamoya disease. Anesth Analg 1997;85:1060-5.

17. Sato K, Shirane R, Kato M, Takashi Y. Effect of inhalational anesthesia on cerebral circulation in Moyamoya disease. J Neurosurg Anesthesiol 1999;11:25-30.

18. Adachi K, Yamamoto Y, Kameyama E, Suzuki H, Horinouchi T. Early postoperative complications in patients with Moyamoya disease: A comparison of inhaled anesthesia with total intravenous anesthesia (TIVA). Masui 2005;54:653-7.

19. Kurehara K, Ohnishi H, Touho H, Furuya H, Okuda T. Cortical blood flow response to hypercapnia during anaesthesia in Moyamoya disease. Can J Anaesth 1993;40:709-13.

20. Nishimoto A, Onbe H, Ueta K. Clinical and cerebral blood flow study in Moyamoya disease. Acta Neurol Scand 1983;60:360-8.

21. Kuwabara Y, Ichiya Y, Sasaki M, Yoshida T, Masuda K, Matsushima T, et al. Response to hypercapnia in moyamoya disease. Cerebrovascular response to hypercapnia in pediatric and adult patients with moyamoya disease. Stroke 1997;28:701-7.

22. Sato K, Shirane R, Yoshimoto T. Perioperative factors related to the development of ischemic complications in patients with moyamoya disease. Childs Nerv Syst 1997;13:68-72.

23. Iwama $\mathrm{T}$, Hashimoto $\mathrm{N}$, Yonekawa $\mathrm{Y}$. The relevance of hemodynamic factors to perioperative ischemic complications in childhood moyamoya disease. Neurosurgery 1996;38:1120-5.

24. Kurokawa T, Chen YJ, Tomita S, Kishikawa T, Kitamura K. Cerebrovascular occlusive disease with and without the moyomoya vascular network in children. Neuropediatrics 1985;16:29-32.

25. Ahn HJ, Kim JA, Lee JT, Kim HS, Shin HJ, Chung IS, et al. Effect of preoperative skull block on pediatric moyamoya disease. J Neurosurg Pediatr 2008;2:37-41.

How to cite this article: Sharma VB, Prabhakar H, Rath GP, Bithal PK. Anaesthetic management of patients undergoing surgery for Moyamoya disease - our institutional experience. J Neuroanaesthesiol Crit Care 2014;1:131-6.

Source of Support: Nil, Conflict of Interest: None declared. 